\title{
The Space of Countably Simple Bounded Functions with Values in a DF-Space
}

\author{
S. DÍAZ, A. FERNÁNDEZ, M. FLORENCIO and P.J. PAÚL
}

ABSTRACT. We study the posibility of lifting some properties, as being a (barrelled, quasi-barrelled, bornological or ultrabornological) $D F, g D F$ or quasi-normable space, from a locally convex space $E$ to the space $S_{\aleph_{0}}(\mu, E)$, of countably-valued and bounded (classes of $\mu$-a.e. equal) functions from a measure space $(\Omega, \Sigma, \mu)$ into $E$.

Let $(\Omega, \Sigma, \mu)$ be a measure space and $E \neq\{0\}$ be a Hausdorff locally convex space. Denote by $S_{\aleph_{0}}(\Sigma, E)$ the space of all functions $\varphi: \Omega \longrightarrow E$ that can be written as

$$
\varphi(\cdot)=\sum_{n=1}^{\infty} \chi s_{n}(\cdot) x_{n}
$$

This research has been supported by La Consejeria de Educacion y Ciencia de la Junta de Andalucia and was presented at the III Conference on Function Spaces held in Poznan (Poland) in 1992.

1991 Mathematics Subject Classification: 46A04, 46A08, 46E30 and 46E40

Editorial Complutense. Madrid, 1994. 
where $\left(x_{n}\right)$ is a bounded sequence from $E$ and $\left(S_{n}\right)$ is a sequence of non-empty and pairwise disjoint subsets of $\Sigma$ covering $\Omega$. A function of this form is called a countably simple bounded function. If we endow $S_{\aleph_{0}}(\Sigma, E)$ with the uniform convergence topology and identify functions that are equal $\mu$-a.e., we obtain the quotient space $S_{\aleph_{0}}(\mu, E)$. This paper is devoted to the possibility of lifting some properties from $E$ to $S_{\mathrm{N}_{0}}(\mu, E)$, mainly in the case when $E$ is a $D F$-space. For the space $C B(X, E)$ of continuous bounded functions, similar results to our Theorems 1-4 here were obtained by Bierstedt, Bonet and Schmets in [2], and for the space $L^{\infty}(\mu, E)$ of essentially bounded measurable functions by Fernández and Florencio in [6]. We refer the reader to the monographs of Jarchow [8] and Pérez Carreras and Bonet [10] for the terminology used in this paper.

To fix some notation, denote by $\mathcal{Q}(E)$ the family of all continuous seminorms defining the topology of $E$, by $\mathcal{U}(E)$ the family of all absolutely convex and closed zero-neighbourhoods of $E$ and by $\mathcal{B}(E)$ the family of all absolutely convex and closed bounded subsets of $E$. Then, a fundamental system of seminorms for the topology defined on $S_{\aleph_{0}}(\Sigma, E)$ is given by the mappings

$$
\bar{q}\left(\sum_{n=1}^{\infty} \chi s_{n} \cdot x_{n}\right)=\sup \left\{q\left(x_{n}\right): n \geq 1\right\},
$$

where $q$ runs through the set $\mathcal{Q}(E)$.

When $\mu$ is the cardinal measure, both spaces $S_{\aleph_{0}}(\Sigma, E)$ and $S_{N_{0}}(\mu, E)$ coincide. In particular, if $\mu$ is the cardinal measure on the set $\mathrm{N}$ of all positive integers, we have that $S_{\aleph_{0}}(\mu, E)=\ell^{\infty}(E)$, the space of all bounded sequences from $E$. Thus, the case studied here can be considered a generalization of this space of vector-valued sequences. We shall see, however, that when $\mu$ is atomless the behaviour can be very different (see Theorem 5 below).

We start by proving that $S_{\aleph_{0}}(\mu, E)$ is a Hausdorff quotient of $S_{\aleph_{0}}(\Sigma, E)$.

Proposition 1. Let $\mathcal{N}_{\mu}$ be the subspace of all functions in $S_{\mathcal{N}_{0}}(\Sigma, E)$ that are equal $\mu$-a.e. to the zero function. Then $\mathcal{N}_{\mu}$ is closed. 
Proof. Let $\varphi$ be a countably simple bounded function such that $\varphi \notin \mathcal{N}_{\mu}$. Then, we can write $\varphi$ as in $(*)$, requiring in addition that $\mu\left(S_{1}\right)>0$ and $x_{1} \neq 0$. Thus, there is a continuous seminorm $q \in \mathcal{Q}(E)$ such that $q\left(x_{1}\right)>0$. Consider the following open neighbourhood of $\varphi$,

$$
V:=\left\{\psi \in S_{\aleph_{0}}(\Sigma, E): \tilde{q}(\psi-\varphi)<q\left(x_{1}\right) / 2\right\}
$$

We only have to show that $V \cap \mathcal{N}_{\mu}$ is empty. Take $\psi \in V$. According to $(*), \psi$ can be written as $\psi=\sum_{m=1}^{\infty} \chi_{T_{m}} y_{m}$. Consider the element of $\Sigma$ defined by

$$
T:=\bigcup_{m \in I} T_{m}, \quad \text { where } I:=\left\{m \in \mathbf{N}: y_{m} \neq 0\right\} .
$$

Since $\psi \neq 0, T$ is non-empty. Let us see that $\mu\left(S_{1} \backslash T\right)=0$. Indeed, if we suppose that $\mu\left(S_{1} \backslash T\right)>0$, then $S_{1} \backslash T$ is non-empty. In this set, $\psi-\varphi$ takes the value $-x_{1}$. Therefore $q\left(x_{1}\right) / 2>\tilde{q}(\psi-\varphi) \geq q\left(x_{1}\right)$ and this is a contradiction. Since $\mu\left(S_{1}\right)>0$, there must be an index $m_{0} \in I$ such that $\mu\left(S_{1} \cap T_{m_{0}}\right)>0$, thus $\mu\left(T_{m_{0}}\right)>0$. Since $y_{m_{0}} \neq 0$, we conclude $\psi \notin \mathcal{N}_{\mu}$.

To avoid trivial cases, we assume the following condition $(C)$ :

(C) There is a sequence $\left(\Delta_{n}\right)$ of pairwise disjoint sets in $\Sigma$ with $\mu\left(\Delta_{n}\right)>0$, for all $n \in \mathbf{N}$.

We follow a common habit and do not distinguish by notation between a map and its $\mu$-equivalence class. In particular, using (*) above and condition (C), for a non-zero element $\varphi \in S_{\mathrm{K}_{0}}(\mu, E)$ we can always choose a representative of the form $\sum_{n=1}^{\infty} \chi S_{n} x_{n}$, where $\left(x_{n}\right)$ is a bounded sequence from $E$ and $\left(S_{n}\right)$ is a pairwise disjoint sequence of subsets of $\Sigma$ with positive measure convering $\Omega$. The set $R(\varphi):=\left\{x_{n}: n \in \mathbf{N}\right\}$ is clearly well-defined, we call it the essential range of the function $\varphi$. The quotient topology of $S_{\aleph_{0}}(\mu, E)$ can be defined by the family of seminorms $q_{\infty}$ given by

$$
\begin{aligned}
q_{\infty}(\varphi): & =\inf \{\tilde{q}(\psi): \psi=\varphi(\mu-\text { a.e. })\} \\
& =\sup \{q(x): x \in R(\varphi)\} \quad(q \in \mathcal{Q}(E)) .
\end{aligned}
$$


We give now some technical results that describe the behaviour of those subsets of $S_{\aleph_{0}}(\mu, E)$ that are lifted from sets in $E$ in a natural way.

If $A$ is a subset of $E$, we say that a function $\varphi \in S_{\mathbb{N}_{0}}(\mu, E)$ takes its values essentially in $A$ if $R(\varphi) \subset A$. We denote by $L(A)$ the subset of all functions in $S_{\mathrm{N}_{0}}(\mu, E)$ that take their values essentially in $A$. The set $L(A)$ inherits certain properties from $A$. We list some of them that will be useful and can be easily checked:

(1) For all subsets $A, B$ of $E$ we have that $L(A) \subset L(B)$ if and only if $A \subset B$.

(2) $L\left(\cap A_{n}\right)=\cap L\left(A_{n}\right)$, for every sequence $\left(A_{n}\right)$ of subsets of $E$.

(3) $L(\alpha A)=\alpha L(A)$ for all $A \subset E$ and scalars $\alpha$.

(4) If either $A$ or $B$ is bounded, then $L(A)+L(B)=L(A+B)$.

(5) $L(A)$ is absolutely convex if so is $A$.

(6) $U \subset E$ is a zero-neighbourhood in $E$ if and only if $L(U)$ is a zero-neighbourhood in $S_{\aleph_{0}}(\mu, E)$. The system $\left\{L(U): U^{\prime} \in \mathcal{U}(E)\right\}$ is a basis of zero-neighbourhoods for the topology of $S_{\aleph_{0}}(\mu, E)$.

(7) $A$ is a bounded subset of $E$ if and only if $L(A)$ is a bounded subset of $S_{\aleph_{0}}(\mu, E)$. Moreover, for every bounded subset $C$ of $S_{\aleph_{0}}(\mu, E)$, there exists $A \in \mathcal{B}(E)$ such that $C \subset L(A)$; just take $A$ to be the closed absolutely convex hull:

$$
A=\overline{\operatorname{acx}}\left(\bigcup_{\varphi \in C} R(\varphi)\right) .
$$

From the list above, it is clear that many properties - like the existence of a fundamental sequence of bounded subsets, the metrizability of the bounded subsets or the countable boundedness property - are equivalent for the spaces $E$ and $S_{\aleph_{0}}(\mu, E)$.

Theorem 1. $S_{N_{0}}(\mu, E)$ is a $D F$-space (resp, a gDF-space) if and only if $E$ is a $D F$-space (resp. a $g D F$-space). 
Proof. $(\Leftarrow)$ Assume that $E$ is a $D F$-space and let $\left(B_{n}\right)$ be an increasing fundamental sequence of absolutely convex bounded subsets in $E$. As we pointed out above, $\left(L\left(B_{n}\right)\right)$ is a fundamental sequence of absolutely convex bounded subsets in $S_{\mathrm{K}_{0}}(\mu, E)$. Now, we have to prove that $S_{\aleph_{0}}(\mu, E)$ is countably-quasi-barrelled. Let $\left(W_{n}\right)$ be a sequence of absolutely convex zero-neighbourhoods in $S_{\aleph_{0}}(\mu, E)$ such that $W=\cap_{n} W_{n}$ is bornivorous. We have to see that $W$ is also a zero-neighbourhood. For every $n \in \mathbf{N}$, take $r_{n}>0$ with $r_{n} L\left(B_{n}\right) \subset 2^{-(n+1)} W$. Then we have

$$
\bigcup_{n \geq 1}\left(r_{1} L\left(B_{1}\right)+r_{2} L\left(B_{2}\right)+\cdots+r_{n} L\left(B_{n}\right)\right) \subset \frac{1}{2} W
$$

Since $\left(W_{n}\right)$ are zero-neighbourhoods in $S_{\aleph_{0}}(\mu, E)$, there exists a sequence $\left(V_{n}\right)$ in $\mathcal{U}(E)$ such that $L\left(V_{n}\right) \subseteq \frac{1}{2} W_{n}$, for all $n \in \mathbf{N}$. Consider, for $n=1,2, \ldots$, the absolutely convex zero-neighbourhoods in $E$ given by

$$
U_{n}:=r_{1} B_{1}+r_{2} B_{2}+\cdots+r_{n} B_{n}+V_{n}
$$

It is clear that $U=\cap_{n} U_{n}$ is bornivorous in the $D F$-space $E$. Therefore $U$ is a zero-neighbourhood in $E$. Finally, we will show that $L(U) \subset W$.

$$
\begin{aligned}
L(U) & =L\left(\bigcap_{n=1}^{\infty} U_{n}\right)=\bigcap_{n=1}^{\infty} L\left(U_{n}\right)=\bigcap_{n=1}^{\infty} L\left(\sum_{k=1}^{n} r_{k} B_{k}+V_{n}\right) \\
& =\bigcap_{n=1}^{\infty}\left(\sum_{k=1}^{n} r_{k} L\left(B_{k}\right)+L\left(V_{n}\right)\right) \subseteq \bigcap_{n=1}^{\infty}\left(\frac{1}{2} W+\frac{1}{2} W_{n}\right) \\
& \subseteq \bigcap_{n=1}^{\infty} W_{n}=W .
\end{aligned}
$$

$\Leftrightarrow$ Now, assume that $S_{\aleph_{0}}(\mu, E)$ is a $D F$-space. If $\left(C_{n}\right)$ is a fundamental sequence of absolutely convex bounded subsets of $S_{\aleph_{0}}(\mu, E)$, we can find a sequence of absolutely convex bounded subsets $\left(B_{n}\right)$ in $E$ such that $C_{n} \subset L\left(B_{n}\right)$ for all $n \in \mathbf{N}$. It is easy to see that $\left(B_{n}\right)$ is an fundamental sequence of bounded sets in $E$. To show that $E$ is countably-quasi-barrelled, let $\left(U_{n}\right)$ be a sequence of absolutely convex 
zero-neighbourhoods in $E$ such that $U=n_{n} U_{n}$ is bornivorous. Then $\left(L\left(U_{n}\right)\right)$ is a sequence of zero-neighbourhoods in $S_{\aleph_{0}}(\mu, E)$ such that $L(U)=\cap_{n} L\left(U_{n}\right)$ is bornivorous. Since $S_{\aleph_{0}}(\mu, E)$ is a $D F$-space, it follows that $L(U)$ is a zero-neighbourhood in $S_{\aleph_{0}}(\mu, E)$, hence $U$ is a zero-neighbourhood in $E$. This finishes the proof.

To prove that $S_{\aleph_{0}}(\mu, E)$ is a $g D F$-space if and only if $E$ is a $g D F$ space use the fact that for all sequences $\left(B_{n}\right)$ in $\mathcal{B}(E)$ and $\left(U_{n}\right)$ in $\mathcal{U}(E)$ we have that

$$
L\left(\bigcap_{n \geq 1}\left(B_{n}+U_{n}\right)\right)=\bigcap_{n \geq 1}\left(L\left(B_{n}\right)+L\left(U_{n}\right)\right),
$$

together with condition $[8,12.3 .1]$. We leave the details to the reader.

Theorem 2. $S_{\aleph_{0}}(\mu, E)$ is quasi-normable if and only if $E$ is quasinormable.

Proof. $(\Leftrightarrow)$ Given any zero-neighbouthood $W$ in $S_{\aleph_{0}}(\mu, E)$, there is an absolutely convex zero-neighbourhood $U$ in $E$ with $L(U) \subset W$. By hypothesis, we can find $V \in \mathcal{U}(E)$ such that for every $\varepsilon>0$ there exists $B \in \mathcal{B}(E)$ with $V \subset B+\varepsilon U$. Then

$$
L(V) \subset L(B+\varepsilon U)=L(B)+\varepsilon L(U) \subset L(B)+\varepsilon W,
$$

so $S_{\aleph_{0}}(\mu, E)$ is quasi-normable.

$(\Rightarrow)$ On the other hand, given $U \in U(E)$, since $L(U)$ is a zero-neighbourhood in $S_{\mathbb{N}_{0}}(\mu, E)$, by hypothesis there exists $V \in \mathcal{U}(E)$ such that for every $\varepsilon>0$ there is $B \in \mathcal{B}(E)$ with $L(V) \subset L(B)+\varepsilon L(U)=$ $L(B+\varepsilon U)$. It follows that $V \subset B+\varepsilon U$, and the proof is finished.

We now study when the space $S_{\aleph_{0}}(\mu, E)$ is quasi-barrelled or bornological for $E$ a $D F$-space. In the characterization of the quasi-barrelled and bornological spaces $\ell^{\infty}(E)$ given by Bierstedt and Bonet in [1, Thm. 5 and Cor. 8] the dual density condition and the strong dual density condition, introduceed and studied by them in the same paper, play an 
essential role. We shall see that these conditions are also essential in our more general case.

These conditions read technically as follows (see [1, Prop. 1.4(b)]): A $D F$-space $E$ with a fundamental sequence $\left(B_{n}\right)$ of bounded subsets, verifies the dual density condition (resp. strong dual density condition) if and only if for every decreasing sequence $\left(\lambda_{n}\right)_{n \geq 1}$ of positive real numbers, there exists $U \in \mathcal{U}(E)$ such that for every $n \geq 1$, we can find $m \geq n$ and $\varepsilon_{n}>0$ with

$$
B_{n} \cap \varepsilon_{n} U \subseteq \overline{\operatorname{acx}}\left(\bigcup_{k=1}^{m} \lambda_{k} B_{k}\right) \quad\left(\operatorname{resp} \cdot B_{n} \cap \varepsilon_{n} U \subseteq \operatorname{acx}\left(\bigcup_{k=1}^{m} \lambda_{k} B_{k}\right)\right)
$$

We also know that a $D F$-space satisfies the dual density condition if and only if its bounded subsets are metrizable [1, Thm. 1.5].

Theorem 3. Let $(\Omega, \Sigma, \mu)$ be a measure space and $E$ be a $D F$ space. Then, the following assertions are equivalent:

(1) E satisfies the dual density condition or, equivalently, each bounded subset of $E$ is metrizable.

(2) $S_{\aleph_{0}}(\mu, E)$ is quasi-barrelled.

Proof. (1) $\Rightarrow(2)$ By Theorem $1, S_{\mathbb{N}_{0}}(\mu, E)$ is a $D F$-space whose bounded subsets are metrizable by properties (6) and (7) above. The implication follows from a well-known result on $D F$-spaces $[9, \S 293 .(12)]$.

$(2) \Rightarrow(1)$ Consider an increasing fundamental sequence $\left(B_{n}\right)$ of closed absolutely convex bounded subsets of $E$, and suppose that (1) does not hold. By reading the dual density condition in the technical form given above, we can see that there exists a decreasing sequence $\left(\lambda_{n}\right)$ of strictly positive numbers such that for every $U \in \mathcal{U}(E)$, we can find $n \geq 1$ with the property that for every $m \geq n$ and every $\varepsilon>0$, in particular $\varepsilon=1$, we have

$$
B_{n} \cap U \not \subset C_{m}:=\overline{\operatorname{acx}}\left(\lambda_{1} B_{1} \cup \lambda_{2} B_{2} \cup \cdots \cup \lambda_{m} B_{m}\right)
$$

This gives us an increasing sequence $\left(C_{m}\right)$ of closed absolutely convex bounded subsets of the $D F$-space $E$ such that: 
(i) $\left(\lambda_{m}^{-1} C_{m}\right)$ is a fundamental sequence of bounded sets in $E$.

(ii) For each $U \in \mathcal{U}(E)$, there is $k \geq 1$ with $B_{k} \cap U \not \subset C_{m}$ for all $m \geq 1$.

We now adapt a technique due to S. Dierolf (see [5, Prop. 4.5]) to get a contradiction. Since every bounded set in $S_{\mathbb{N}_{0}}(\mu, E)$ is absorbed by some $L\left(C_{n}\right)$, the set

$$
W=\bigcup_{n \geq 1} L\left(C_{n}\right)
$$

is bornivorous in $S_{\aleph_{0}}(\mu, E)$. Then, the closure $\bar{W}$ of $W$ in $S_{\aleph_{0}}(\mu, E)$ is a bornivorous barrel in $S_{\aleph_{0}}(\mu, E)$, and by hypothesis, it is a zeroneighbourhood in $S_{\aleph_{0}}(\mu, E)$. Then, there exists $U \in \mathcal{U}(E)$ such that

$$
L(U) \subseteq \frac{1}{2} \vec{W}
$$

Now, by (ii) there is a bounded sequence $\left(x_{n}\right)$ in $U$ such that $x_{n} \notin C_{n}$. Since $\left(C_{n}\right)$ are closed subsets of $E$, there is a sequence $\left(V_{n}\right) \subset \mathcal{U}(E)$ such that

$$
x_{n} \notin C_{n}+V_{n}, n \in \mathbf{N} .
$$

By (i), and since the sequence $\left(C_{n}\right)$ is increasing, the set

$$
V=\bigcap_{k \geq 1}\left(\frac{1}{2} C_{k}+V_{k}\right)
$$

is 'bornivorous in the $D F$-space $E$. Hence, $V$ is a zero-neighbourhood in $E$ and $L(V)$ is a zero-neighbourhood in $S_{\mathrm{k}_{0}}(\mu, E)$. We have that

$$
\begin{aligned}
L(U) & \subset \frac{1}{2} \bar{W} \subset \frac{1}{2} W+L(V)=\bigcup_{n \geq 1} \frac{1}{2} L\left(C_{n}\right)+L(V) \\
& =\bigcup_{n \geq 1} \frac{1}{2} L\left(C_{n}\right)+\bigcap_{n \geq 1}\left(\frac{1}{2} L\left(C_{n}\right)+L\left(V_{n}\right)\right) \\
& \subset \bigcup_{n \geq 1}\left(L\left(C_{n}\right)+L\left(V_{n}\right)\right)=\bigcup_{n \geq 1} L\left(C_{n}+V_{n}\right) .
\end{aligned}
$$


Clearly, the function $\varphi: \Omega \rightarrow E$ defined by $\varphi=\sum_{n>1} x_{n} \chi_{\Delta_{n}}$, where $\left(\Delta_{n}\right)$ is the sequence given by condition $(C)$, is in $S_{\aleph_{0}}(\mu, E)$. Moreover, $\varphi \in L(U)$ because the bounded sequence $\left(x_{n}\right)$ is contained in $U$. By $(* * *)$, there is $n \in \mathbf{N}$ such that $\varphi \in L\left(C_{n}+V_{n}\right)$. Since $\mu\left(\Delta_{n}\right)>0$, we have that $x_{n} \in C_{n}+V_{n}$, which is in contradiction with $(* *)$.

Theorem 4. Let $(\Omega, \Sigma, \mu)$ be a measure space and $E$ a $D F$-space. Then, the following assertions are equivalent:

(1) E satisfies the strong dual density condition.

(2) $S_{\aleph_{0}}(\mu, E)$ is bornological.

Proof. Let $\left(B_{n}\right)$ be an increasing fundamental sequence of absolutely convex closed bounded subsets of $E$. To prove that $(2) \Rightarrow(1)$, suppose that (1) does not hold. Reading the strong dual density condition in the technical form given above, we have that there exists a decreasing sequence $\left(\lambda_{n}\right)$ of strictly positive numbers such that for each $U \in \mathcal{U}(E)$, we can find $n \geq 1$ with the property that for every $m \geq n$ and $\varepsilon>0$, in particular $\varepsilon=1$, we have

$$
B_{n} \cap U \not \subset C_{m}:=\operatorname{acx}\left(\lambda_{1} B_{1} \cup \lambda_{2} B_{2} \cup \cdots \cup \lambda_{m} B_{m}\right) .
$$

This gives us an increasing sequence $\left(C_{m}\right)$ of absolutely convex bounded subsets of $E$. Take $W=\cup_{n} L\left(C_{n}\right)$. This set $W$ is absolutely convex and bornivorous in $S_{\aleph_{0}}(\mu, E)$. Since $S_{\aleph_{0}}(\mu, E)$ is bornological, then $W$ is a zero-neighbourhood, and we can find $U \in \mathcal{U}(E)$ such that $L(U) \subset W$.

Since $B_{n} \cap U \not \subset C_{m}$, we can take $x_{m} \in\left(B_{n} \cap U\right) \backslash C_{m}$ for all $m \geq 1$. If we set $\varphi=\sum_{m=1}^{\infty} x_{m} \chi \Delta_{m}$, where $\left(\Delta_{m}\right)$ is the sequence from condition $(C)$, then $\varphi \in S_{\aleph_{0}}(\mu, E)$. Moreover, $\varphi \in L(U) \subset W=\cup_{n} L\left(C_{n}\right)$. Therefore, there exists $n_{0} \in N$ such that $\varphi \in L\left(C_{n_{0}}\right)$. Since $\mu\left(\Delta_{n_{0}}\right)>0$, then $x_{n_{0}} \in C_{n_{0}}$ and this is a contradiction with the selection of the $x_{n}{ }^{\prime} s$.

(1) $\Rightarrow(2)$ Since $\left(L\left(B_{n}\right)\right)$ is an increasing fundamental sequence of absolutely convex closed bounded subsets in $S_{\aleph_{0}}(\mu, E)$ and this is a $D F$ space, we only have to show that if $W$ is an absolutely convex bornivorous subset of $S_{\aleph_{0}}(\mu, E)$, then $W \cap L\left(B_{n}\right)$ is a zero neighbourhood 
in $L\left(B_{n}\right)$ for every $n \in \mathbf{N}$, when $L\left(B_{n}\right)$ is endowed with the topology inherited from $S_{\aleph_{0}}(\mu, E)$.

Since $W$ is bornivorous, there exists a decreasing sequence of positive real numbers $\left(\lambda_{n}\right)$ such that $\lambda_{n} L\left(B_{n}\right) \subset W$ for every $n \in \mathbf{N}$.

Bearing in mind the definition of the strong dual density condition, there exists $U \in \mathcal{U}(E)$ such that from all $n \in \mathbf{N}$, we can find $m \geq n$ and $\varepsilon_{n}>0$ with

$$
B_{n} \cap \varepsilon_{n} U \subset \operatorname{acx}\left(\frac{\lambda_{1}}{2} B_{1} \cup \frac{\lambda_{2}}{2^{2}} B_{2} \cup \cdots \cup \frac{\lambda_{m}}{2^{m}} B_{m}\right) .
$$

Therefore,

$$
\begin{aligned}
L\left(B_{n}\right) \cap \varepsilon_{n} L(U) & =L\left(B_{n} \cap \varepsilon_{n} U\right) \subset L\left(\operatorname{acx}\left(\bigcup_{k=1}^{m} \frac{\lambda_{k}}{2^{k}} B_{k}\right)\right) \\
& \subset L\left(\sum_{k=1}^{m} \frac{\lambda_{k}}{2^{k}} B_{k}\right)=\sum_{k=1}^{m} \frac{\lambda_{k}}{2^{k}} L\left(B_{k}\right) \subset W .
\end{aligned}
$$

Finally, $L\left(B_{n}\right) \cap \varepsilon_{n} L(U) \subset W \cap L\left(B_{n}\right)$ for every $n \in \mathbf{N}$, so the proof is finished.

To study when $S_{\aleph_{0}}(\mu, E)$ is barrelled or ultrabornological, we shall use the abstract results given in [3] (barrelledness) and in [4] (ultrabornology) for a locally convex space endowed with a suitable Boolean algebra of projections. A family $P_{\Sigma}=\left\{P_{S}: S \in \Sigma\right\}$ of continuous linear projections in $E$ is called an $(\Omega, \Sigma, \mu)$-Boolean algebra of projections if the following conditions are satisfied:

(i) $P_{\Omega}$ is the identity on $E$.

(ii) $P_{S}=0$ whenever $S \in \Sigma$ and $\mu(S)=0$.

(iii) $P_{S \cap T}=P_{S} \cdot P_{T}$ for all $S, T \in \Sigma$.

(iv) $P_{S \cup T}=P_{S}+P_{T}$ for all disjoint $S, T \in \Sigma$. 
The results mentioned above can be stated as follows. (Similar results as in [3] and [4] for some spaces of (scalar or vector-valued) continuous functions defined on an interval $[a, b] \subset \mathbf{R}$, have been obtained independently and about the same time by Gilioli [7].)

Theorem A. ([3, Cor. 1 and 2] and [4, Cor. 1 and 2].) Let $(\Omega, \Sigma, \mu)$ be a $\sigma$-finite measure space. Let $E$ be a Hausdorff locally convex space and $P_{\Sigma}$ be an $(\Omega, \Sigma, \mu)$-Boolean algebra of projections. Assume that $P_{\Sigma}$ is equicontinuous and that the following conditions holds:

(•) If $\left(\Omega_{n}\right)$ is a decreasing sequence in $\Sigma$ with $\mu\left(\cap_{n} \Omega_{n}\right)=0,\left(x_{n}\right)$ is a bounded sequence in $E$ such that every $x_{n}$ is supported in $\Omega_{n}$ (i.e. $\left.P_{\Omega_{n}}\left(x_{n}\right)=x_{n}\right)$, and $\left(\alpha_{n}\right)$ is a sequence in $\ell^{1}$, then the series $\sum_{n} \alpha_{n} x_{n}$ converges in $E$.

Then we have:

(1) If $E$ is quasi-barrelled and $P_{S}(E)$ is barrelled for each atom $S \in \Sigma$, then $E$ is barrelled.

(2) If $E$ is quasi-barrelled and $\mu$ is atomless, then $E$ is barrelled.

(3) If $E$ is bornological and $P_{S}(E)$ is ultrabornological for each atom $S \in \Sigma$, then $E$ is ultrabornological. gical.

(4) If $E$ is bornological and $\mu$ is atomless, then $E$ is ultrabornolo-

To use Theorem A in our case, we state the following lemma.

Lemma. For every subset $S \in \Sigma$, denote

$$
P_{S}: \varphi \in S_{\aleph_{0}}(\mu, E) \rightarrow P_{S}(\varphi)=\chi_{S} \cdot \varphi \in S_{\aleph_{0}}(\mu, E)
$$

Then we have:

(1) The set $\left\{P_{S}: S \in \Sigma\right\}$ is an equicontinuous Boolean algebra of projections on $S_{\aleph_{0}}(\mu, E)$.

(2) If $S$ is an atom, then $P_{S}\left(S_{\aleph_{0}}(\mu, E)\right)$ is isomorphic to $E$. 
(3). $S_{\aleph_{0}}(\mu, E)$ satisfies the condition $(\bullet)$ in Theorem A, i.e. if $\left(\Omega_{n}\right)$ is a decreasing sequence of subsets of $\Sigma$ such that $\mu\left(\cap_{n} \Omega_{n}\right)=0$ and $\left(\varphi_{n}\right)$ is a bounded sequence in $S_{\aleph_{0}}(\mu, E)$ such that $\varphi_{n}$ is supported in $\Omega_{n}$, for each $n \in \mathbf{N}$ and if $\left(\alpha_{n}\right)$ is a sequence from $\ell_{1}$, then the series $\sum_{n} \alpha_{n} \varphi_{n}$ converges in $S_{\aleph_{0}}(\mu, E)$.

Proof. (1) The algebraic part is easy. To prove the equicontinuity, simply note that $P_{S}(L(U)) \subset L(U)$, for every $U \in \mathcal{U}(E)$ and $S \in \Sigma$.

(2) It is enough to prove that any function of $S_{\aleph_{0}}(\mu, E)$ is constant on $S$. In this case, the isomorphism is the natural. Suppose that $\varphi \in S_{\aleph_{0}}(\mu, E)$ is not constant on $S$. 'Then, there exist $x_{1}, x_{2} \in E$ with $x_{1} \neq x_{2}$, such that the subsets

$$
S_{1}=\left\{\omega \in S: \varphi(\omega)=x_{1}\right\} \quad \text { and } \quad S_{2}=\left\{\omega \in S: \varphi(\omega)=x_{2}\right\}
$$

are disjoint and they have positive measure. Since $S$ is an atom, we have that $\mu\left(S_{1}\right)=\mu\left(S_{2}\right)=\mu(S)$ and we get a contradiction.

(3) Since $\left(\Omega_{n}\right)$ is decreasing and each $\varphi_{n}$ is supported in $\Omega_{n}$, it follows that the series $\sum_{n} \alpha_{n} \varphi_{n}$ converges pointwise $\mu$-a.e. to a function $\varphi$ because outside every $\Omega_{n}$ there is only a finite number of non-zero terms and $\mu\left(\cap_{n} \Omega_{n}\right)=0$. Finally, note that $\varphi$ is countably simple and bounded because

$$
R(\varphi) \subset \bigcup_{n=1}^{\infty}\left\{\sum_{k=1}^{n} \alpha_{k} x_{k}, x_{k} \in R\left(\varphi_{k}\right), k \in \mathbf{N}\right\}
$$

is bounded and countable, and that $\varphi$ is also the limit in $S_{\aleph_{0}}(\mu, E)$ of $\sum_{n} \alpha_{n} \varphi_{n}$.

From this lemma and Theorem A, we have the following.

Theorem 5. Let $(\Omega, \Sigma, \mu)$ be a $\sigma$-finite measure space and $E$ a DF-space.

(a) If the measure $\mu$ has atoms, then

(1) $S_{\aleph_{0}}(\mu, E)$ is barrelled if and only if $E$ is barrelled and each bounded subset of $E$ is metrizable. 
(2) $S_{\mathrm{N}_{0}}(\mu, E)$ is ultrabornological if and only if $E$ is ultrabornological and $E$ satisfies the strong dual density condition.

(b) If the measure $\mu$ is atomless, then

(1) $S_{N_{0}}(\mu, E)$ is barrelled if and only if each bounded subset of $E$ is metrizable.

(2) $S_{\mathbb{N}_{0}}(\mu, E)$ is ultrabornological if and only if $E$ satisfies the strong dual density condition.

Acknowledgements. We want to thank the referee for his remarks and for calling our attention to references [2] and [7].

\section{References}

[1] Bierstedt. K.D. and Bonet, J.: Dual density conditions in (DF) spaces I. Resultate Math., 14 (1988), 242-274.

[2] Bierstedt, K.D., Bonet, J. and Schmets, J.: (DF)-spaces of type $C B(X, E)$ and $C V(X, E)$. Note Mat., 10 (suppl. n.1) (1990), 127-148.

[3] Díaz, S., Fernández, A., Florencio, M. and Paúl, P.J.: An abstract Banach-Steinhaus theorem and applications to function spaces. Resultate Math., 23 (1993), 242-250.

[4] Díaz, S., Fernández, A., Florencio, M. and Paúl, P.J.: A wide class of ultrabornological spaces of measturable functions. J. Math. Anal. Appl. (to appear).

[5] Dierolf, S.: On spaces of continuous linear mappings between locally convex spaces. Note Mat., 5 (1985), 147-225.

[6] Fernández, A. and Florencio, M.: The space of essentially bounded measurable functions with values in a DF-space. Proc. Roy. Irish Acad., 93A (n.1) (1993), 87-95.

[7] Gilioli, A.: Natural ultrabornological, non-complete, normed function spaces. Arch. Math. (Basel), 61 (1993), 465-477. 
[8] Jarchow, H.: Locally Convex Spaces. B.G. Teubner, Stuttgart, 1981.

[9] Köthe, G.: Topological Vector Spaces. I. Springer-Verlag, Berlin, Heidelberg, New York, 1969.

[10] Pérez Carreras, P. and Bonet, J.: Barrelled locally Convex Spaces. Elsevier North-Holland Publ. Co., Amsterdam, New York, Oxford, 1987.

E.S. Ingenieros Industriales,

Avda. Reina Mercedes $5 / n$,

41012-Sevilla

SFAIN
Recibido: 7 de enero de 1993

Revisado: 1 de junio de 1993 